\title{
Interfacial Oil Droplets
}

\author{
Xue Hua Zhang and William Ducker* \\ Department of Chemical and Biomolecular Engineering and Particulate Fluids \\ Processing Centre, University of Melbourne, Melbourne 3010, Australia
}

Supporting data 
Figure S1. AFM image of the interface between OTS-Silicon and a 25\% ethanol aqueous solution. No decane is added to the solution, and no droplets are observed on the solid.

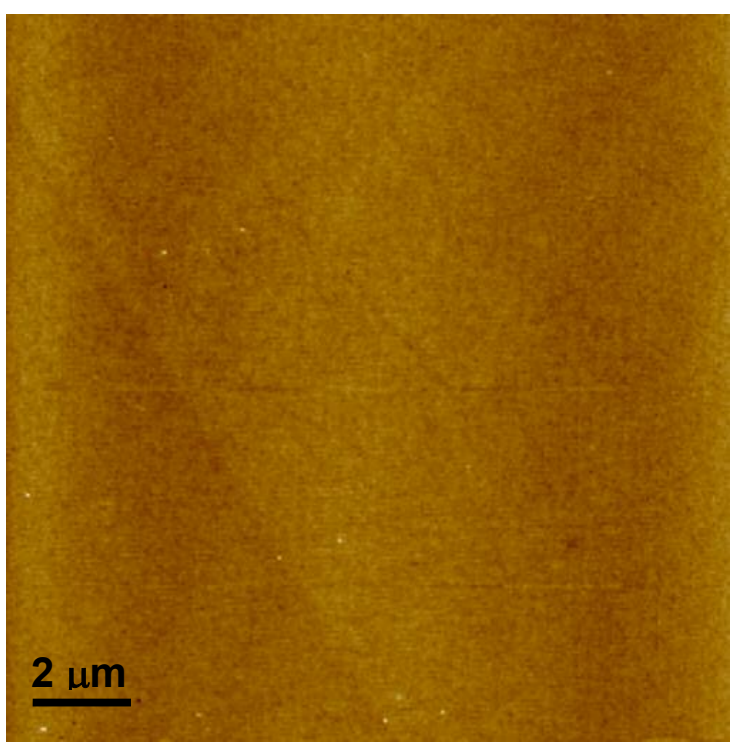


Figure S2. Change in volume of the droplets as a function of the height.

Figure 7 shows that the droplets decrease in volume with time. The following figure shows the magnitude of decrease in volume from $80 \mathrm{~min}$ to $120 \mathrm{~min}$ as a function of the measured droplet height at $80 \mathrm{~min}$ for a sample of droplets in the images in Figure 7. The volume of the droplet was calculated from the radius of the three phase line and the height with the assumption that the droplets are spherical caps. There is significant noise in the data, but there is a trend showing that higher droplets have a greater decrease in volume.

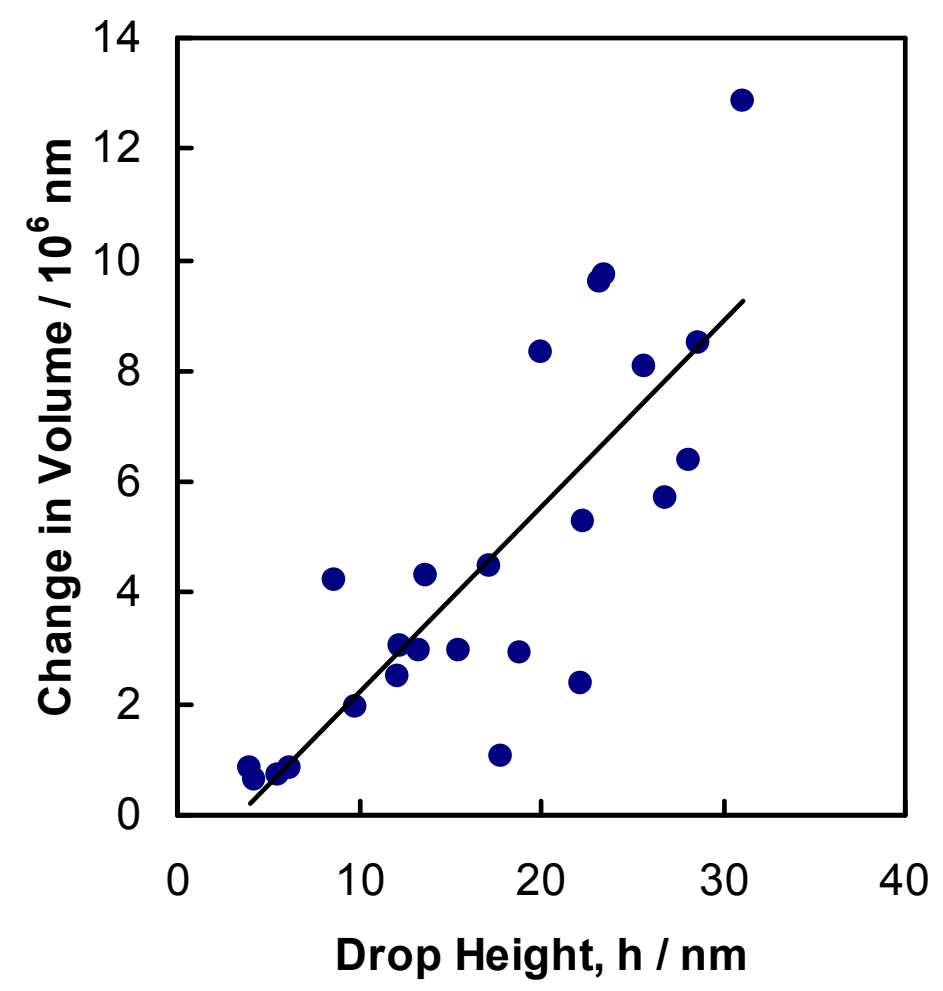

